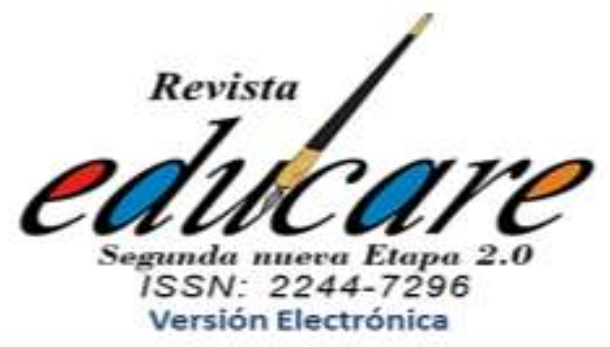

Volumen 25 N$^{\circ} 1$ Enero - Abril 2021

$(457-474)$

\title{
EL ADULTO MAYOR Y LA EDUCACIÓN NO FORMAL EN TIEMPOS DE PANDEMIA
}

Aura Rosa Dávila de Velásquez *

ORCID: https://orcid.org/0000-0002-8264-1930

Universidad Pedagógica Experimental Libertador

(Venezuela)

\author{
THE ELDERLY ADULT AND NON-FORMAL \\ EDUCATION IN TIMES OF PANDEMIC
}

* Licda. en Administració Esp. en Finanzas Docente Universitario y Tutor Académico UBA Doctorando en Educación UPEL. aurarosadavilav@gmail.com

\section{Recibido: \\ 25-01-2021 \\ Aceptado: \\ 01-04-2021}



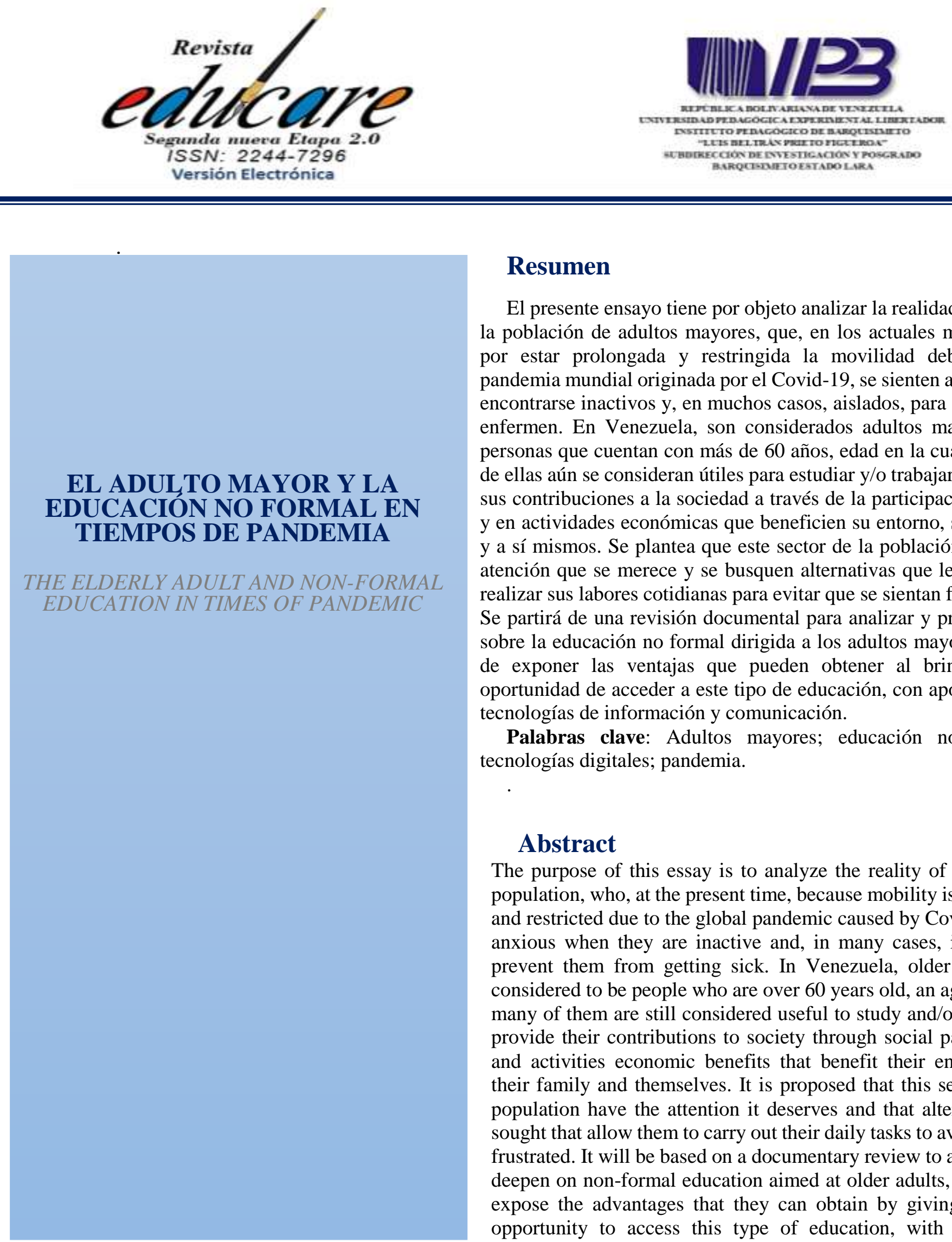

\title{
Resumen
}

El presente ensayo tiene por objeto analizar la realidad que vive la población de adultos mayores, que, en los actuales momentos, por estar prolongada y restringida la movilidad debido a la pandemia mundial originada por el Covid-19, se sienten ansiosos al encontrarse inactivos y, en muchos casos, aislados, para evitar que enfermen. En Venezuela, son considerados adultos mayores las personas que cuentan con más de 60 años, edad en la cual muchas de ellas aún se consideran útiles para estudiar y/o trabajar y brindar sus contribuciones a la sociedad a través de la participación social y en actividades económicas que beneficien su entorno, su familia y a sí mismos. Se plantea que este sector de la población tenga la atención que se merece y se busquen alternativas que le permitan realizar sus labores cotidianas para evitar que se sientan frustrados. Se partirá de una revisión documental para analizar y profundizar sobre la educación no formal dirigida a los adultos mayores, a fin de exponer las ventajas que pueden obtener al brindarles la oportunidad de acceder a este tipo de educación, con apoyo en las tecnologías de información y comunicación.

Palabras clave: Adultos mayores; educación no formal; tecnologías digitales; pandemia.

\begin{abstract}
The purpose of this essay is to analyze the reality of the elderly population, who, at the present time, because mobility is prolonged and restricted due to the global pandemic caused by Covid-19, feel anxious when they are inactive and, in many cases, isolated, to prevent them from getting sick. In Venezuela, older adults are considered to be people who are over 60 years old, an age at which many of them are still considered useful to study and/or work and provide their contributions to society through social participation and activities economic benefits that benefit their environment, their family and themselves. It is proposed that this sector of the population have the attention it deserves and that alternatives be sought that allow them to carry out their daily tasks to avoid feeling frustrated. It will be based on a documentary review to analyze and deepen on non-formal education aimed at older adults, in order to expose the advantages that they can obtain by giving them the opportunity to access this type of education, with support in information technologies and communication.
\end{abstract}

Keywords: Older adults; non-formal education; digital technologies: pandemic 


\section{Introducción}

El año 2020 transformó la vida de las personas por las consecuencias de la pandemia mundial, un año de aprendizaje para muchos donde se le da gran importancia a la vida por medio del distanciamiento social y la práctica de medidas de bioseguridad. Los adultos mayores han sido las personas más vulnerables al contagio del virus originado por el Covid19, pero si aunado a ello, no se les brinda apoyo para mantener una vida activa dentro de las posibilidades que éstos puedan tener, la frustración puede ocasionarles profundos problemas psicológicos y de salud. Rivoir y Morales (2019) indican, "las personas de 60 años o más constituyen el grupo de edad de mayor crecimiento y su proporción con respecto a la población total está aumentando, lo que hace que cada vez su peso como grupo poblacional sea también mayor." (pp.51-52), hecho que debe sensibilizar a la sociedad en general, prestándoles la atención que se merecen para brindarles una longevidad de calidad.

Los adultos mayores son considerados un valioso aporte para la sociedad, por ser las personas que por su experiencia, responsabilidad y compromiso coadyuvan a que las economías de los países prosperen, lo que convierte a muchos de ellos, en un activo significativo con expectativas claras al momento de tomar decisiones. Asimismo, este sector de la población se ha empoderado de tal manera, que, a través de la participación social buscan soluciones a problemas individuales y colectivos, lo que los impulsa a aprender cosas nuevas que agreguen valor a sus vidas.

Hay adultos mayores que se encuentran saludables o ya están totalmente recuperados luego de contagiarse por el coronavirus, que tienen el deseo de seguir contribuyendo a la sociedad y brindar sus habilidades, capacidades y destrezas en su hogar o en el medio donde se desenvuelven. Mogollón (2012) indica: “Las personas de edad deben tener la oportunidad de trabajar hasta que quieran y sean capaces de hacerlo en el desempeño de trabajos satisfactorios, productivos y de seguir teniendo acceso a la educación y a los programas de capacitación”. (p.57) De igual manera, la Constitución de la República Bolivariana de Venezuela (1999) garantiza una educación integral, permanente y de calidad, dentro del principio de igualdad, sin que exista ningún tipo de limitaciones para acceder a ella, puesto que las aptitudes, vocación y aspiraciones se pueden construir a partir del proceso de enseñanza-aprendizaje al cual tienen acceso las personas; por lo que, debe prevalecer la educación para todos y a lo largo de toda la vida. 
En tal sentido, si los adultos mayores muestran interés en seguir de alguna manera dinámicos y acceder a algún tipo de aprendizaje o realizar algún trabajo que los haga sentirse útiles, debe dárseles la oportunidad de lograrlo sin necesidad de acudir a un aula de clase o a un determinado sitio de trabajo, lo pueden hacer desde sus hogares como una opción para mantenerse activos y motivados al gozar de un buen estado de salud, mental y físico.

En ese mismo orden de ideas, Sarrate (2002) citado en Moreno (2015), plantea "Se trata de un nuevo paradigma fundamentado en el principio de que la educación debe estar presente desde el inicio hasta el final del ciclo vital de la persona." (p.115) El ser humano tiene derecho a recibir una educación durante toda su vida, es decir una educación permanente que le permita desarrollar sus capacidades y actitudes para adaptarse a los cambios constantes que se le presenten. En el presente ensayo, se aborda la educación no formal; al respecto, Cárdenas(2018),opina "El concepto de Educación No Formal también se puede expresar paralelamente en términos de educación recurrente, cuando se refiere como una educación para toda la vida, o también como educación alternante, cuando hace referencia a la educación complementaria."(p.33) Así, el individuo no solo debe tener garantizada la educación formal, sino ir más allá, al incorporar conocimientos a su vida a través de la educación no formal y hacerlo de manera complementaria como el medio que le permita acceder al trabajo, ampliar sus capacidades o sencillamente, estar ocupados al llevar a cabo actividades de su gusto para sentirse útiles y orgullosos de sí mismos.

En los últimos tiempos han cambiado los escenarios para la educación, adaptándose a nuevas formas de enseñanza con apoyo en las tecnologías de información y comunicación, y la educación no formal no escapa de ello, pues el acceso a las nuevas tecnologías, permiten romper con las barreras temporales y espaciales para aprender haciendo sin necesidad de asistir a un salón de clases. En el desarrollo de este ensayo se profundizará sobre la educación no formal como una alternativa de aprendizaje dirigida a los adultos mayores, así como la importancia para este sector de la población de acceder a las tecnologías digitales como medio para adquirir nuevos conocimientos que les haga sentirse activos y útiles. 


\section{Desarrollo}

\section{Educación No Formal: Una alternativa para los adultos mayores}

Los sucesos acaecidos en el mundo a partir de la pandemia derivada del Covid-19, ha controlado la movilidad de las personas, las actividades de las empresas y la educación presencial; además, ha transformado la actuación de muchas personas, en especial la de los adultos mayores. Noriega, García y Torres (s/f) citado en Castillo y Olivares (2017) opinan:

Con la edad aumentan las crisis, los problemas, los factores estresantes cobran mayor importancia; y se agravan por las difíciles situaciones de vida que presentan muchas personas ancianas, y también a causa de la disminución de las fuerzas físicas para hacer frente a esas dificultades. (p.14)

Los individuos al llegar a esa etapa de adultez, experimentan cambios emocionales, físicos y afectivos que inciden directamente en su actuación, cambios que asociados a circunstancias como las actuales, producto de la pandemia mundial, podrían desencadenar enfermedades psicosomáticas que generen angustia a estas personas y a su círculo familiar. Muchos adultos mayores han permanecido en sus hogares por largo tiempo, limitados a seguir instrucciones para preservar su salud, unos, profesionales, otros no; algunos de ellos, sin realizar ningún tipo de actividad que los haga sentirse útiles, activos o capaces de resolver los problemas que ocurren en su entorno.

Es por lo planteado, que a este sector de la población se le debe brindar la oportunidad de realizar actividades que los mantenga ocupados, lejos de los inconvenientes originados por la pandemia, y una manera de lograrlo, es facilitarle el acceso a algún tipo de formación que les permita incorporar nuevos conocimientos a sus vidas y mantener vigentes los principios de educación: aprender a conocer, aprender a hacer, aprender a ser y aprender a convivir. Al respecto, Guillén (2008), explica lo importante que es para el individuo comprender estos principios:

-Aprender a conocer: es el medio y finalidad de la vida humana. -Aprender a hacer: está estrechamente vinculado a cómo enseñar al alumno a poner en práctica sus conocimientos y, al mismo tiempo, como adaptar la enseñanza al futuro mercado de trabajo. -Aprender a ser: se refiere a la libertad de pensamiento, de juicio, de 
sentimiento y de imaginación que necesitan para que sus talentos alcancen la plenitud y seguir siendo artífices, en la medida de lo posible, de su destino. -Aprender a convivir: centrado en el aprendizaje del individuo a vivir juntos, a vivir con los demás, evitar los conflictos y conocerse a sí mismo y conocer a los demás, a su cultura y espiritualidad. (p.146)

En tal sentido, la persona aprende a conocer, al interactuar con sus semejantes y utilizar el sentido crítico y reflexivo de acuerdo a sus capacidades para comprender lo que ocurre en el entorno que lo rodea; aprende a hacer, cuando toma las decisiones más acertadas para dar respuesta a sus problemas, al considerar sus experiencias adquiridas, sus ideas creativas; aprende a ser, cuando actúa por propia convicción, a crecer como ser humano y desarrollar un pensamiento crítico sin dejarse influenciar por el medio; y, aprende a convivir, para tener la capacidad de compartir y aceptar a sus semejantes, admitir y conciliar a través del diálogo las diferencias que puedan existir, ser solidarios para trabajar en equipo y dar solución a los problemas comunes.

Dichos principios también están presentes en la educación no formal y son elementos importantes en la formación de los adultos mayores para garantizarles una mejor calidad de vida. Este tipo de educación está estrechamente relacionado a la andragogía, pues el individuo es responsable de su propio aprendizaje, ya que asume los cambios que se producen en su vida por los conocimientos previos que posee y por la interacción que existe en su entorno. Villalobos y Riveros (2018), indican que las premisas sobre el aprendizaje en adultos, giran en torno a los siguientes factores:

1) Aprenden de manera distinta a los niños, por lo cual las técnicas de enseñanza deben ser también distintas. 2) Valoran un aprendizaje significativo basado en su propia experiencia y que les ayude a encontrar soluciones prácticas a sus problemáticas y necesidades más sentidas. 3) La relación entre quienes facilitan este interaprendizaje y el grupo participante es más horizontal, desapareciendo la noción de un conocimiento superior y una relación de poder basada en la propiedad y acceso a tal conocimiento. (p.24)

En el aprendizaje para adultos, en especial el dirigido a las personas de la tercera edad, la metodología de enseñanza debe estar encaminada a cumplir con sus expectativas de vida, pues las experiencias personales y las habilidades o capacidades que poseen, facilitan 
su aprendizaje, impulsándolos a través de la motivación y el deseo de superación a poner en práctica nuevas ideas que aporten soluciones a los problemas que se les presenten. Como se expuso en líneas anteriores, este tipo de aprendizaje tiene sus bases en la andragogía.

Precisamente, Morales y Leguizamón (2018) describen las premisas de la teoría andragógica, expuesta porMalcolm Knowles, como la necesidad de saber por qué las personas deben aprender algo; se refiere al auto concepto del adulto como ser responsable de su vida y de sus decisiones, un ser comprometido con su formación y el valor de la experiencia y cómo esta incide en su personalidad, así como, la disposición para aprender y la orientación de dicho aprendizaje vinculadas al compromiso y al crecimiento personal. Asimismo, la motivación agrega valor a los adultos que quieren ser exitosos, tienen control sobre su aprendizaje y hacen que sea placentero.(pp.166-167)En efecto, la andragogía surge como una alternativa para los adultos mayores, puesto que, al estar dispuestos a aprender por su propia voluntad, lo hacen motivados a conocer cosas nuevas, al dirigir su aprendizaje con un gran compromiso y responsabilidad y seguros de que sus vivencias aportarán los beneficios que desean encontrar en este tipo de enseñanza.

Malcolm Knowles (2006), citado en Castillo (2018) y considerado el padre de la Andragogía por sus aportes en torno a este tipo de aprendizaje, indicaba que la andragogía "es un conjunto de principios fundamentales sobre el aprendizaje de adultos que se aplica a todas las situaciones de tal aprendizaje.” (p.65) Los adultos mayores tienen muy claro lo que desean y hacia dónde dirigirse, por tener sus propios requerimientos y expectativas para enriquecer sus conocimientos a lo largo de su vida. Es por ello, que la educación no formal está llamada a cubrir las necesidades de formación de este sector de la población, lo que redundará en bienestar y felicidad para ellos. Barba y Salamanca (2019), sostienen:

La educación no formal está sustentada en un conjunto de actividades educativas, organizadas, sistemáticas y planificadas en tiempos y espacios variables, dirigidas a grupos determinados de población, que no están directamente encaminados a la obtención de título, la cual es realizada fuera del marco del sistema oficial de enseñanza, por ser una oferta voluntaria, planificada, pero flexible y con diversidad de métodos y contenidos, que pareciera ser una propuesta educativa más prescindible. (p. 226) 
La educación no formal rompe con el paradigma de la rigidez de la educación formal, plantea métodos y contenidos organizados y sistemáticos, pero más flexibles y adaptados a las necesidades de sus participantes. Esta modalidad de enseñanza surge como una alternativa para todos aquellos adultos mayores que desean formarse en un oficio determinado, ya sea para ocupar su tiempo libre, prestar sus servicios a su comunidad o simplemente, resolver sus problemas domésticos y así, contribuir de alguna manera a la sociedad. Al respecto, Lloret (2017) expresa, que, al estar situada fuera del sistema de la enseñanza reglada, facilita ciertas tendencias metodológicas por no tener que impartir unos currículos estandarizados; además, por no ser obligatoria, facilita la posibilidad de métodos y estructuras organizativas más flexibles, participativas, adaptables a los usuarios y a las necesidades específicas de cada quien. (p.12)

De igual forma, la educación no formal permite que los participantes de manera autónoma y espontánea accedan a ella para interactuar y mostrar sus necesidades inmediatas sin ningún tipo de imposición social. Este tipo de instrucción le proporciona al adulto mayor las herramientas necesarias para tomar sus propias decisiones al utilizar sus conocimientos previos, lo que favorece su desarrollo personal, mejora la autoestima y el sentido de superación hacia lo que más le agrada; además, de potenciar sus habilidades y capacidades hacia la creatividad y disponer del tiempo y el espacio necesarios para alcanzar sus expectativas de vida y su participación en los problemas propios de su familia y su comunidad.

En ese mismo orden de ideas, Cárcamo (2008) citado en Ormeño (2016), expresa que la educación no debe considerarse como un proceso limitado en el tiempo y en el espacio, ni confiar solo en la escuela y en la asistencia continua de los alumnos o participantes, debe ir más allá con la educación no formal, donde el aprendizaje es auto dirigido, con responsabilidad, autodeterminación y motivación. (p.90) Los tiempos han cambiado y con ellos la educación, pues se ha promovido nuevas formas de educar a través del aprendizaje no formal al utilizar como apoyo las nuevas tecnologías de información y comunicación, para superar las brechas digitales al permitirse el acceso a todos por igual.

Igualmente, Amorós (2013) expresa, "La razón de la educación no formal surge como una necesidad de la educación ante el desarrollo constante de innovación tecnológica, haciendo que la sociedad requiera formas flexibles y permanentes de educación". (p.3) Este 
tipo de educación es a corto plazo y no amerita la presencialidad en el aula, puesto que se dicta a distancia o virtual, lo que resulta más cómodo para los que a ella acceden; el autoaprendizaje, la responsabilidad, la dedicación y la creatividad, son armas esenciales que debe poseer el individuo para lograr incorporar nuevos conocimientos a su vida de manera permanente y dar respuesta a los desafíos que se le presenten.

Por otra parte, de acuerdo a proyecciones del Instituto Nacional de Estadística INE (2011), citado por Bravo y Lamus (2019), Venezuela está experimentando un proceso de envejecimiento poblacional que para el año 2018, se situó con un porcentaje de personas de 60 años y más cercano al 10\% y con una esperanza de vida de 77,18 para las mujeres y de 71,22 años para los hombres, siendo uno de los primeros países que presenta un considerable incremento de población adulta mayor en la región. (p.149). Sin embargo, producto de la pandemia, esta es la población en el país que más ha sufrido los embates por las consecuencias del coronavirus, por lo que es necesario prestarle mayor atención para evitar que la depresión, la inactividad y las noticias que día a día conocen, les genere más angustia, ansiedad o estrés. Una forma, es abrirles la posibilidad de realizar cursos o talleres que se encuentran en la red o que son ofertados por instituciones privadas o gubernamentales de forma virtual o a distancia, muchos de ellos gratuitos, donde puedan desarrollar sus competencias con autonomía y flexibilidad para mantener un envejecimiento activo.

Al hacer referencia al envejecimiento activo, Martínez, González y otros (2018), indican que la Organización Mundial de la Salud adoptó este término para desvincular a las personas mayores con la pasividad, discapacidad e inactividad social y laboral y considerarlas altamente proactivas, con participación de poder y de necesitar hacer, lo que invalida las creencias prejuiciosas que en ocasiones se tienen hacia este sector tan importante de la población. (p.63.) De allí, la importancia de dirigir la mirada hacia los adultos mayores para favorecer el potencial que aún poseen y que puedan realizar las actividades que más les atraen, lo que les permite mantener una vida prolongada con salud y bienestar.

Da Silva y Scortegagna (2015) argumentan sobre la existencia de instituciones, inclusive universidades, preocupadas por el envejecimiento poblacional, que ofrecen alternativas en el área de la educación para acoger a las personas de la tercera edad y brindarles herramientas con conocimientos e información que los actualicen, favoreciendo su mayor participación en la sociedad. (p.7) Estos espacios educativos empoderan de alguna 
manera a este segmento de la población, dándoles la oportunidad de hacerse sentir como personas que aún tienen mucho por aportar a sus familias, a la comunidad a la cual pertenecen y por supuesto, a su país. Asimismo, existen organizaciones gubernamentales que ofertan la educación no formal, no solo a jóvenes sino también a adultos mayores, como una alternativa para aprender nuevos oficios, este es el caso del Instituto Nacional de Capacitación y Educación Socialistas INCES.

Es por lo planteado, que en los actuales momentos cuando aún se mantiene el distanciamiento social producto de la pandemia mundial y que son los adultos mayores los más afectados, la educación No Formal se vislumbra como una opción para que este sector de la población acceda a ella para ocupar su tiempo libre al realizar un oficio de su preferencia, lo que redundará, en una mejor calidad de vida.

\section{Las tecnologías digitales y el aprendizaje en los adultos mayores}

No se puede ocultar que los adultos mayores hasta hace algún tiempo era la población más desfavorecida al acceso de las computadoras y al uso de Internet, pues mantenían una situación de dependencia para dar solución a sus problemas cotidianos; pero esta condición está cambiando, ya que en la actualidad, son muchos los adultos mayores que disponen de un teléfono inteligente o algún otro dispositivo digital para realizar consultas o transacciones bancarias, buscar y obtener información de interés, enterarse de las noticias a través de las redes, realizar compras, conocer algunas aplicaciones telefónicas que les ayuden a dar solución a algunos problemas, o simplemente, socializar con otras personas.

Los adultos mayores para adaptarse a los cambios que han surgido en los últimos tiempos y mantener su equilibrio emocional, no se han quedado atrás en el uso de las tecnologías digitales. Vega y Quintero (2019) señalan que en el mundo globalizado que se vive no se puede ocultar el uso intensivo de la información y el conocimiento y el creciente aumento de los adultos mayores, donde el Estado, la Academia y la Tecnología deben propiciar las condiciones adecuadas para que éstos gocen de una vida activa en la que se contempla el acceso a las tecnologías digitales. (p.136), lo que se transforma en una gran ventaja para ellos, pues les brinda la oportunidad de mantenerse comunicados constantemente y la facilidad de incorporarse a algún tipo de educación y labores que los mantenga activos. 
Las personas de la tercera se están adaptando al acceso y uso de Internet, formando parte poco a poco de su cotidianidad, puesto que se mantienen informados y socialmente activos al interactuar con otras personas, en especial con sus seres queridos que se encuentran en otras fronteras, lo que los impulsa a su vez, a la capacitación. Es el momento de que el Estado, conjuntamente con las instituciones gubernamentales, las ONG, la Academia, entes tecnológicos y hasta el entorno familiar, realicen un mayor esfuerzo para hacer llegar a la mayor parte de este segmento de la población la oportunidad de alfabetizarse tecnológicamente, permitiéndoles la inclusión a un mundo que cada día se va transformando, lo que redundará en salud, mejorando su estado de ánimo y, por ende, su calidad de vida.

Es cierto que los adultos mayores por no haber nacido en la era digital requieren de mayor capacitación en torno a las tecnologías de información y comunicación; sin embargo, la motivación, la seguridad y la confianza que se pueda depositar en ellos, les ayudará a vencer las barreras que puedan encontrar al enfrentarse al mundo tecnológico, perdiendo el miedo de todo aquello que para ellos era desconocido, lo que, sin lugar a dudas, les aportará una mayor suma de bienestar. Según Peral, Arenas y Villarejo (2015) citado en Porras (2018),

Los factores psicológicos explican el desarrollo de competencias y habilidades en los mayores que favorecerá la utilización de las redes sociales, y que permitirá optimizar y prolongar su empleo a medida que envejecen, puesto que suponen un instrumento de comunicación que permitirá alcanzar niveles de bienestar y beneficios para el cuidado de la salud y mejora de la autosuficiencia. (p.19)

A pesar que no se puede generalizar cuando se habla de los adultos mayores, por existir barreras sociales, culturales, económicas, geográficas, entre otras, que marcan la diferencia entre unos y otros, la incidencia de acceder a las nuevas tecnologías por parte de este segmento de la población es cada día mayor, dado que les facilita la interacción con otras personas y los motiva a su formación personal, teniendo la oportunidad también de incrementar sus capacidades y habilidades para tomar sus propias decisiones y dar solución a los problemas que puedan presentarse en su entorno. Por su parte, Ormeño (2016), señala:

En este contexto nos encontramos con una sociedad proactiva, influenciada por las tecnologías digitales y la globalización, en que debe procurar visualizar las bondades que le aportan para alcanzar un estado de bienestar y donde todos los ciudadanos 
puedan obtener y compartir información independientemente del lugar o momento en el que se encuentren, constituyendo un elemento clave para el desarrollo económico y el bienestar social. (p.29)

A raíz de la pandemia, las tecnologías digitales han impactado inesperadamente en todos los niveles de educación de los ciudadanos del mundo, y poco a poco han tenido que acostumbrarse a no dejarlas de lado y obviar las limitaciones personales, geográficas y temporales que puedan existir. Casamayou y Morales (2017), se refieren a la heterogeneidad de situaciones e intereses de las personas mayores, aduciendo que son sujetos sociales con derecho a ejercer su ciudadanía, así como contribuir y participar activamente en los procesos sociales democráticos donde no se les estigmatice. (p.217) Por ello, los aportes de los adultos mayores a la sociedad no deben ser menospreciados, pues mientras ellos tengan la voluntad, el ímpetu, el coraje y la motivación para seguir activos y productivos, habrá que apoyarlos para hacerles sentir que son importantes para su nación.

Por su parte, Ormeño (ob.cit.),se refiere al aprendizaje invisible como el nuevo cambio social y tecnológico que apunta hacia la reestructuración de los sistemas educativos centrados en el profesor y en un salón de clases, a un sistema educativo que satisfaga las expectativas del individuo, donde se pueda trabajar a distancia y que el facilitador sea el orientador del proceso educativo. (p.101) Precisamente, este aprendizaje persigue crear conciencia en el individuo para impulsarse a través de la motivación y fortalecer cualidades como la responsabilidad, la toma de decisiones, el autocontrol y la actitud, por ser elementos fundamentales para su autoaprendizaje, sin necesidad de copiar normas o reglas impuestas por otras personas y utilizar como apoyo las tecnologías digitales.

Asimismo, Barrantes y Cozzubo (2015) citado en Rivoir y Morales (2019) “comprueban el aprendizaje intergeneracional en el vínculo entre menores de edad y personas mayores dentro del hogar. Confirman el incremento del uso de Internet cuando conviven otras personas mayores". (p.299). El hecho que adultos mayores estén rodeados de niños y jóvenes en su hogar, favorece el uso de las tecnologías digitales en aquéllos, logrando incentivarse en la socialización con otras personas, conocer novedades en la web, hasta el hecho de impulsarse a tomar algunos cursos de interés.

De Benedictis, Camel y otros (2019) indican que, apoyadas en las nuevas tecnologías, las universidades pueden ofrecer la educación no formal para capacitar a todo aquel que desee 
hacerlo, inclusive como complemento de los planes de estudio formales, a través de cursos, foros, seminarios, talleres, entre otros, lo que posibilita la actualización de los profesionales en los campos de su desempeño y propicia el mejoramiento permanente. (p.61). En Venezuela, a raíz de la pandemia, ha crecido el número de universidades que ofrece cursos a distancia a cualquier tipo de población, algunos de forma gratuita, hecho que ha abierto las puertas a todos aquellos adultos mayores que desean formarse para el trabajo o incorporar nuevos aprendizajes a sus vidas; todo ello, como una manera de hacerlos sentir útiles, lo que, sin lugar a dudas, les proporcionaría un gran equilibrio emocional.

El adulto mayor al familiarizarse con la web, podrá tener acceso a un sinnúmero de cursos, talleres, foros, diplomados, sin limitaciones de ningún tipo. Asimismo, no hay que olvidar la ventaja que existe con la plataforma YouTube, donde se muestran videos que pueden facilitar el aprendizaje en cualquiera de los oficios que desee conocer, que además de proporcionar satisfacción personal, puede representar un ingreso monetario para él, si así se lo propone, sin necesidad de desplazarse a ningún lugar y en la disponibilidad de horario y tiempo que determine.

Existen en el país centros de capacitación para el trabajo y asociaciones civiles sin fines de lucro que imparten la educación no formal. El Instituto Nacional de Capacitación y Educación Socialistas INCES (2021) ofrece en la actualidad, cursos en línea gratuitos en diferentes áreas en todo el territorio nacional, dirigidos a personas de cualquier edad. Estos cursos son a corto plazo y pueden acceder todas aquellas personas que deseen adquirir conocimientos o ampliarlos en determinados oficios, obteniendo al final del mismo, un certificado con la fecha del curso, su duración y el contenido cubierto, instrumento que le permite obtener un empleo sin necesidad de tener experiencia laboral.

La educación no formal apoyada en las tecnologías digitales, ha llegado a la vida de los adultos mayores como una alternativa de aprendizaje muy justificada, para ayudarles a cambiar el panorama que hasta los momentos han tenido, producto del confinamiento por la pandemia mundial. Por este motivo, para acceder a este tipo de educación, es indispensable que aquellos adultos mayores que no manejen las tecnologías digitales, cuenten con la asistencia y paciencia de sus hijos, nietos y amigos más cercanos. De esta forma, el estar ocupados, distraídos con alguna actividad que les guste, les proporcionará tranquilidad y bienestar, aspectos que se traducen en salud y longevidad. 


\section{Cierre}

La educación no formal, concebida como una formación no institucionalizada y flexible en relación con la educación formal, está cada día más presente en la vida de los ciudadanos, debido al progreso continuo de las tecnologías digitales implícitas en su manejo, que ofrecen múltiples alternativas de aprendizaje a los que deseen acceder a ella. Además, sirve como complemento de la educación formal, para ocupar el tiempo libre, capacitarse para el trabajo o aprender algún oficio que permita a los adultos mayores brindar aportes en su entorno familiar o en la comunidad a la cual pertenecen y así, tener la oportunidad de envejecer sintiéndose saludables, útiles, activos y comprometidos con la sociedad.

Los adultos mayores necesitan la atención de todos, sin excepción, no solo por las consecuencias que la pandemia ha traído al mundo, sino por el hecho de ser las personas más sensibles por su envejecimiento natural que incide directamente en su salud y por las carencias afectivas o emocionales que puedan tener. Ellos, en su mayoría, son personas que desean contribuir social, económica y culturalmente con el país, ser partícipes delos avances que se produzcan; de allí, la importancia y el deber de brindarles apoyo para que, desde la tranquilidad de sus hogares y con la ayuda de un computador, puedan acceder a la educación no formal como una alternativa para hacerles sentir útiles, capaces, orgullosos de sí mismos, que aprendan un oficio nuevo o fortalezcan los conocimientos que ya poseen, lo que redundará en beneficios para ellos, su entorno familiar y por supuesto, para la nación; que no signifique una carga, sino todo lo contrario, un agente activo y productivo.

La interacción tecnológica que los más jóvenes de la familia puedan tener con los adultos mayores, les permitirá a éstos, ingresar a un mundo nuevo, rompiendo con los paradigmas y tabúes que tenían al colocarse frente a un computador, sintiéndose agradecidos al aumentar su autoestima y confianza en sí mismos. Es una forma de corresponderles a ese amor incondicional que proporcionaron a su grupo familiar cuando en el pasado necesitaban de ellos; es tratar de hacerles sentir más felices, tomados en cuenta, útiles, al realizar las actividades que más les apasionan; es, en definitiva, prestarles la atención que se merecen para que vivan con la satisfacción de que aportaron algo a la nación hasta el final de sus días. 


\section{Referencias}

Amorós, L. (2013). Inclusión digital en la educación no formal. Edutec, Revista Electrónica de Tecnología Educativa, 45, 14.https://www.edutec.es/revista/index.php/edutec-e/article/view/13/pdf_23

Barba, L. y Salamanca, L. (2019).Hacia un trabajo conjunto entre la educación formaly no formal: Una propuesta para apoyar la inserción de niños, niñas, adolescentes y jóvenes con necesidades educativas diversas. Revista de Educación Social, 29, 219246. https://eduso.net/res/wp-content/uploads/documentos/1249.pdf

Barrantes R. y A. Cozzubo (2015). Edad para aprender, edad para enseñar: el rol del aprendizaje intergeneracional intrahogar en el uso de la internet por parte de los adultos mayores en Latinoamérica. Lima, Departamento de Economía. (Documento de Trabajo 411)

Bravo, M. y Lamus, T. (2019) Atención integral al adulto mayor en Venezuela: un proceso de reflexión desde su recorrido institucional-legal. Revista Arbitrada Interdisciplinaria de Ciencias de la Salud y Vida,3 (5), 146-168. Santa Ana de Coro, Venezuela.https://www.researchgate.net/publication/331928617_Atencion_integral _al_adulto_mayor_en_Venezuela_un_proceso_de_reflexion_desde_su_recorrido_in $\underline{\text { stitucional-legal }}$

Cárcamo L. y Nesbet, F. (2008). Nativos Digitales Chilenses: Los jóvenes, al sur de laInternet. Redalyc.org, 63 (11).https://www.redalyc.org/pdf/819/81912006003.pdf

Cárdenas, O. (2018). La educación no formal en el IPES. Un análisis de los efectosgenerados en el proceso de transformación social de los vendedores informales en Bogotá (2015-2017) (Tesis de grado Maestría en Educación). Universidad Militar Nueva

Granada.https://repository.unimilitar.edu.co/bitstream/handle/10654/18078/C\%C3\% A1rdenasHern\%C3\%A1ndezOmar2018.PDF.pdf? sequence=3\&isAllowed=y

Casamayou, A. y Morales, M. (2017) Personas mayores y tecnologías digitales: desafíos de un binomio. Psicología, Conocimiento y Sociedad, 7(2), 199226.https://www.researchgate.net/publication/321581340_Personas_mayores_y_tec $\underline{\text { nologias_digitales_desafios_de_un_binomio }}$ 
Castillo, C. y Olivares, T. (2017) El adulto mayor y su participación dentro de la educación no-formal, como una forma de inclusión social.(Tesina Licenciado en Psicología).Universidad Academia de Humanismo Cristiano.Chile.http://bibliotecadigital.academia.cl/bitstream/handle/123456789/421 0/TPSICO\%20705.pdf? sequence=1\&isAllowed=y

Castillo, F. (2018). Andragogía, andragogos y sus aportaciones. Revista Voces de la Educación,3(6), 64-76.Universidad Regional del Sureste, Oaxaca, México.https://www.revista.vocesdelaeducacion.com.mx/index.php/voces/article/vi $\underline{\text { ew/120 }}$

Constitución delaRepúblicaBolivarianadeVenezuela(1999). Gaceta Oficial Extraordinaria $\mathrm{N}^{\circ} 36.860$ de fecha 30 de diciembre de 1999.

Da Silva, R. y Scortegagna, P. (2015) Universidad Abierta alas personas adultas mayores: Espacio de Pedagogía Social, Educación y Empoderamiento.Actualidades Investigativas en Educación, 15 (3), 1-18.Universidad de Costa Rica.https://www.scielo.sa.cr/pdf/aie/v15n3/1409-4703-aie-15-03-00505.pdf

De Benedictis, G., Camel, O.; Sequera, N.; Álvarez, E. (2019). La educación no formal: una herramienta importante para la formación del estudiante de medicina. Revista de $\begin{array}{llll}\text { Ciencias } & \text { Médicas } & \text { ARS }\end{array}$ (3).https://www.researchgate.net/publication/331289524_La_educacion_no_formal _una_herramienta_importante_para_la_formacion_del_estudiante_de_medicina

Guillén, J. (2008)Estudio crítico de la obra: La Educación encierra un tesoro. Informe a la UNESCO de la Comisión Internacional sobre la Educación para el Siglo XXI, presidida por Jacques Delors Laurus,14 (26),136-167. Universidad PedagógicaExperimental Libertador, Caracas, Venezuela.https://www.redalyc.org/pdf/761/76111491007.pdf

Instituto Nacional de Capacitación y Educación Socialista (2021). Cursos Inces 2021, PNA, Adultos. Inscripciones. Venezuela.https://www.actualidad24.com/2013/08/requisitos-cursos-estudios-inces.html

Knowles, M. (2006). Andragogía. México: Oxford.

Lloret, B. (2017). Educación No Formal. Los Campamentos de Verano: Una escuelamás.(Trabajo Final de Grado Maestro de Educación Primaria). Universitat 


\section{Jaume}

I.http://repositori.uji.es/xmlui/bitstream/handle/10234/171322/Lloret\%20Colonques \%20Bel\%C3\%A9n_TFG.pdf?sequence=1\&isAllowed=y

Martínez, T.; González, C.; Castellón, G. y González, B. (2018). El envejecimiento, la vejez y la calidad de vida: ¿éxito o dificultad?Revista Finlay, 8 (1), 5965.http://scielo.sld.cu/pdf/rf/v8n1/rf07108.pdf

Mogollón, E. (2012) Una perspectiva integral del adulto mayor en el contexto de la educación.Revista Interamericana de Educación de Adultos, 34 (1),56-74. Centro de Cooperación Regional para la Educación de Adultos en América Latina y el Caribe Pátzcuaro, México. https://www.redalyc.org/pdf/4575/457545090005.pdf

Morales, O. y Leguizamón, M. (2018). Teoría Andragógica: Aciertos y Desaciertos enla Formación Docente en Tic. Revista Praxis \& Saber,9 (19),161181.http://www.scielo.org.co/pdf/prasa/v9n19/2216-0159-prasa-9-19-161.pdf

Moreno-Crespo, P. (2015). Educación a lo largo de la vida: aula de mayores. Revista Fuentes, 17,113-133. http://dx.doi.org/10.12795/revistafuentes.2015.i17.05.

Noriega, M., García, M. y Torres, M. (s/f). Capítulo 2, Proceso de envejecer: cambios físicos, cambios psíquicos y cambios sociales. Universidad de Barcelona.

Ormeño, A. (2016). Uso de las tecnologías digitales en el aprendizaje formal, no formale informal en estudiantes de la carrera de Odontología de la Universidad de Los Andes, Santiago de Chile.(Tesis Doctoral Educación y Sociedad). Universidad de Barcelona.http://hdl.handle.net/10803/402107

Peral-Peral, B., Arenas-Gaitán, J., y Villarejo-Ramos, Á. (2015). De la brecha digital a la brecha psico-digital: Mayores y redes sociales. Revista Comunicar, 23(45).

Porras, A. (2018) TIC, alfabetización digital y envejecimiento satisfactorio: un estudio longitudinal. Universidad de Córdoba.https://helvia.uco.es/bitstream/handle/10396/15902/TFM_Antonio_Jes\%C 3\%BAs_PorrasMoral.pdf?sequence $=1$

Rivoir, A. y Morales, M. (2019) Tecnologías digitales: miradas críticas de la apropiación en América Latina. Libro Digital, 1a ed. Buenos Aires: CLACSO; Montevideo: RIAT. http://biblioteca.clacso.edu.ar/clacso/se/20191128031455/Tecnologias-digitales.pdf 
Sarrate, M. (2002). La educación de adultas, ámbito prioritario de la educación permanente.

En E. López-Barajas Zayas y M.L. Sarrate Capdevila, La educación de personas adultas: reto de nuestro tiempo. Madrid: Dykinson

Vega, O. y Quintero, S. (2019). Persona adulta mayor y TIC: Un ambiente propicio para consolidar. Revista Anales en Gerontología, 11,129-140. Universidad de Costa Rica.https://revistas.ucr.ac.cr/index.php/gerontologia/article/view/34013/41175

Villalobos, O. y Riveros, H. (2018). Modelación de Emprendimientos para Jóvenes Rurales: manual para el diseño e implementación de un ejercicio participativo. Instituto Interamericano de Cooperación para la Agricultura, México.https://www.researchgate.net/publication/327023248_MEJOR_Modelacion _de_Emprendimientos_para_Jovenes_Rurales 